\title{
Design Optimization of MBR System for Oily Wastewater Treatment in
}

\author{
Port \\ Jian-Gong Wang \\ Laboratory of Waterway Environmental Protection Technology, Tianjin Water Transport \\ Engineering Science Research Institute, Tianjin 300456, China \\ w0621@vip.sina.com
}

\begin{abstract}
Keywords: MBR System, Oil-Bearing Wastewater, Cross-Flow Aeration, Biotechnology
Abstract. The optimal design of MBR system for oil film wastewater was introduced. The influence of aeration rate, aeration structure and reactor structure on the average cross flow velocity was studied. The average cross flow velocity between films was $1.25 \sim 1.28 \mathrm{M} / \mathrm{s}$ is a reasonable parameter to effectively slow down membrane fouling. The actual operation of the 12-month project shows that the negative pressure of the membrane module is stable below $0.02 \mathrm{MPa}$. G and does not exceed the critical operating pressure control standard $0.05 \mathrm{Mpa}$ at the time of far chemical cleaning. The MBR system is applied to the oil-pollution of the technical problems for the promotion of MBR system provides a reasonable reference data.
\end{abstract}

\section{Introduction}

Membrane Bioreactor (MBR) is a new type of water treatment system that combines wastewater treatment biotechnology with membrane separation technology. The use of biological methods of pollutants in the sewage has high degradation efficiency and integrated membrane of the efficient interception of the sewage effluent quality has been greatly improved and improved [1, 2]. However, when the MBR treatment process is applied to the oily wastewater treatment of the port, there are some problems such as contaminant pollution and blockage of petroleum contaminants, which will affect the service life of the film and thus affect the water quality Stability and reliability $[3,4]$.

Reasonable MBR system structure design can effectively avoid and slow down the membrane pollution, in which the intermittent flow rate is a key factor in mitigating membrane fouling [5]. In conventional MBR processing system, usually rely on a reasonable aeration structure, the flow to maintain a certain Flow rate $(1 \mathrm{~m} / \mathrm{s}$ or so) to slow down membrane fouling [6]. The use of MBR to deal with oily wastewater in the process, usually need a higher cross-flow rate in order to ensure the life of the diaphragm, which requires the membrane structure of the membrane and the aeration system to optimize the design [7]. Based on the calculation results of Yusuf Christi-Murray Moo Young and the characteristics of oily wastewater, this design presents a new membrane module form, which optimizes the spatial structure of the module and obtains a high flow rate $(1.25 \sim 1.28 \mathrm{~m} / \mathrm{s})$ The film pollution, extended the use of the membrane cycle, to provide MBR system to promote the use of technical data [8].

\section{Project Overview}

The main process flow of the oily wastewater treatment system in a port in the north is shown in Fig.1 Biochemical reaction tank inoculation of sludge using a sewage treatment plant in Hong Kong sewage treatment plant sludge, the sludge after a simple treatment directly into the hydrolysis of 
acidification pool and MBR pool, hydrolytic acidification pool and MBR pool inoculation sludge concentration were: $5000 \mathrm{mg} / \mathrm{L}, 8500 \mathrm{mg} / \mathrm{L}$. After 35 40 days of continuous and stable operation, the effective removal rate of COD is stable at 32\% 40\%, and the removal rate of petroleum (after pretreatment) is more than $70 \%$. , MBR water quality (hydrolysis of acidification tank of the water) in Table 1.

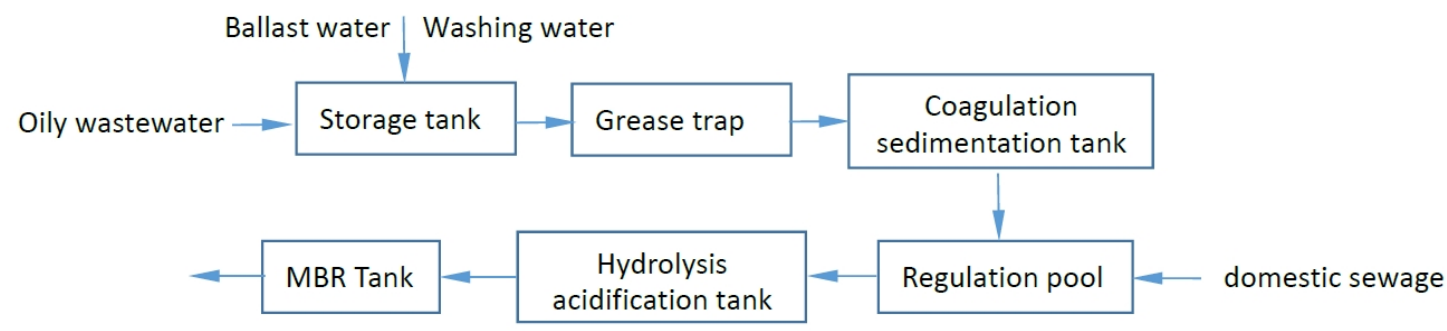

Figure 1 oil-containing wastewater treatment process

Table 1 system operation results

\begin{tabular}{ccccc}
\hline project & $\begin{array}{c}\text { Item Water } \\
(\mathrm{mg} / \mathrm{L})\end{array}$ & $\begin{array}{c}\text { Hydrolysis Cell } \\
\text { Water(mg/L) }\end{array}$ & $\begin{array}{c}\text { MBR Water } \\
(\mathrm{mg} / \mathrm{L})\end{array}$ & $\begin{array}{c}\text { MBR Pool Removal } \\
\text { PerformanceMBR }(\%)\end{array}$ \\
\hline $\mathrm{COD}$ & $420 \sim 2420$ & $258 \sim 1120$ & $30 \sim 44$ & $88.4 \sim 95.7$ \\
BOD $_{5}$ & $104 \sim 613$ & $81 \sim 376$ & $3.5 \sim 9.0$ & $96.8 \sim 97.8$ \\
Petroleum & $40 \sim 84$ & $2.0 \sim 5.2$ & Non detected & - \\
$\mathrm{NH}_{3}-\mathrm{N}$ & $25 \sim 40$ & $15 \sim 22$ & $1.2 \sim 2.9$ & $89.1 \sim 92.2$ \\
Total nitrogen & $35 \sim 50$ & - & $3 \sim 12$ & $76.2 \sim 94.4$ \\
\hline
\end{tabular}

\section{MBR Pool Design Optimization}

The oil-containing wastewater is pretreated through the storage tank, the slanting plate, the coagulation sedimentation, the hydrolysis and acidification, etc., and the MBR reaction cell enters from the top of the hydrolysis acidification tank. It can be seen from Table 1 that the main pollutants in the effluent of the hydrolysis tank are removed to a large extent, but the concentration of various pollutants is still high, and the concentration of petroleum pollutants is still maintained at 2.0 5.2mg/L, Easy to lead to oil adhesion in the MBR wire, to accelerate the contamination of the film wire, affecting the stability of the MBR reactor $[9,10]$.

In order to prolong the service life of the membrane and slow down the pollution rate of the membrane, the surface flow rate model of the oil-containing wastewater membrane system was developed based on the proposed model of the aerosolized liquid flow velocity proposed by Yusuf Christi and Murray Moo Young. And then guide the optimal design of MBR structure.

Designed with an integrated membrane bioreactor, the membrane module is built into a bioreactor and pumped through a self-priming pump to obtain a treated effluent. The integrated membrane bioreactor is divided into two parts, the inlet end and the effluent end of the MBR are formed under the aeration of the bottom to form an internal circulation type stripping reactor. The stripping reactor is composed of a riser zone and Descending zone composition, in the up flow area is usually equipped with aeration device, the drainage area is not, and compared with the former, its low gas content.

(1) Design of Membrane De-aeration Valve

When designing the aeration tubing of the membrane, it is necessary to design a vent pipe on the aeration pipe of each membrane pool and install a vent valve. This design can effectively solve the 
problem of tail pipe run. The main reason for the tail pipe run is the perforation of the perforation of the aeration tube, which causes the air pressure in the aeration tube to rise and the gas from the tail pipe. After the design of the empty tube, when a membrane pool when the opening of the membrane corresponding to the opening of the valve, the rapid release of the air inside the aeration tube, the water quickly into the aeration tube, to restore aeration, the aeration tube water fast, play a positive and negative two-way erosion of the purpose of aeration hole, effectively prevent the hole of the fouling. Ventilation tube design needs to pay attention to the selection of its diameter, aeration pipe is less than DN100, the vent valve and aeration pipe diameter is the same; aeration pipe is larger than DN100, the vent valve than the aeration pipe diameter Small one to two can be.

(2) Design film frame leveling bracket

Uneven frame will lead to uneven aeration, so that the membrane is easy to mud. Film frame was uneven most likely to appear in the membrane frame floor installation. Floor installation, the membrane frame directly installed in the bottom of the ground, due to the construction of the bottom of the roughness is difficult to guarantee, so the membrane frame installed in the bottom is difficult to ensure smooth. In view of this problem, we believe that the design should be designed membrane frame leveling bracket, the membrane frame is no longer installed in the membrane on the ground, but installed in the use of angle made of the bracket, so you can ensure that the film flatness The Or the use of hanging installation.

(3) Water production pipe full pipe design

In order to ensure that the backwash pump at any time from the water mains on the water for backwash, the general is need to ensure the water between the equipment to form a full flow of water. At present, we designed the MBR membrane system of backwash water is basically directly from the water mains on the pumping, if the water mains of the drain than the MBR equipment between the water mains low, the water main pipe is difficult to form a full flow, It is difficult to pump the pump from the water mains. You can design an inverted U-shaped pipe on the mains, man-made water mains to carry out local elevation, to ensure that the MBR equipment within the water mains for the full flow, so that the backwash pump can always pump from the water mains Carry out backwashing.

(4) Bottom of the membrane design drains

Membrane pool in the sludge concentration is high, the bottom of the membrane pool is easy to mud, grit, design drainage can be emptied in the membrane pool when the effective removal of the bottom of the pool sediment and grit, reduce the manual clean-up strength. Drainage ditch design need to pay attention to, drains should be slope to the set wells, the slope of not less than $1 \%$, the location of the drain to avoid the installation of the membrane frame.

(5) Membrane pool level and the height difference between the water pump is not less than 2 meters

Water pump design installation height should be lower than the membrane level 2 meters and above. MBR membrane system using the production pumps are centrifugal pumps, centrifugal pump vertical suction range is generally 4-6 meters, the United States to run MBR film to allow the maximum transmembrane pressure difference of 50kpa (equivalent to 5 meters head), if the membrane pool The level difference between the level and the water pump body is small, or negative, then with the running time, the increase in the pressure of the transmembrane, the water pump may form cavitation cannot produce water. Appropriate increase in the level of the membrane and the height difference between the pump can use the pressure generated by the height difference, reduce the pump water required suction, MBR membrane system to ensure stable water production. 
(6) De-foaming tube design

MBR membrane aeration strength of large, sewage in high-intensity aeration was prone to foam. The production of the foam will affect the operation of the entire membrane environment, is not conducive to observe the operation of the membrane pool. MBR process used in the hollow fiber membrane cannot be exposed to the air for a long time; otherwise the performance of the film will be affected. Membrane pool in the maintenance, the need to emptying the membrane pool, then you can spray through the de-foaming tube to the membrane silk moisturizing, play the role of protective film wire.

(7) Optimization Design of Membrane Pool

In the membrane of the production of water pipes on the addition of a membrane tank and a control valve main purpose is to facilitate the pool of water, simplify the MBR membrane system piping. MBR membrane system for RC cleaning, the need to fill the membrane filled with water. The use of each membrane pool of water production, in which to add a water supply pipe and a control valve, $\mathrm{RC}$ through the backwash pump to backwash the water through the tube to the pool of water, thus simplifying the RC replenishment Piping, making the MBR system more concise.

8) CEB system

MBR system in the long-term operation, the membrane flux will increase with the running time and slow down, take timely chemical cleaning can effectively reduce the flux decline. Chemical cleaning, that is, to remove the membrane surface and membrane pore adsorption of pollutants, to restore membrane flux, to maintain the normal operation of membrane bioreactor. System chemistry to strengthen the backwash is usually used sodium hypochlorite, citric acid two kinds of drugs.

\section{MBR Pool Operation Effect}

The above model is applied to the engineering practice after the verification of the double-layer hollow fiber membrane module of 1: 1 project. The operating parameters of the membrane module are: $10 \mathrm{~L} / \mathrm{m} 2 \cdot \mathrm{h}$ of the membrane module; The suction and stop time ratio is 10min: $2 \mathrm{~min}$; soda ratio of 1:20. MBR has been stable operation for one and a half years, the membrane module operating negative pressure stable below $0.02 \mathrm{MPa}$, much lower than the chemical cleaning when the operating pressure control standard $0.05 \mathrm{Mpa}$, effectively solve the oil pollutants for membrane components pollution, MBR The process is applied to similar projects paving the way.

\section{Acknowledgements}

This work was supported by the Research of "Study of foreign soil water retention, prevent salinization, heavy metal soil remediation, soil backfill and ecological restoration technique in land reclamation. (TKS150218).

\section{References}

[1] Zhu Liang, Zhu Fengchun, Xu Xuchang, et al. Pilot study of MBR/PAC combined process for tailings of sewage plant. China Water Supply and Drainage, 2006, 25 (5), p.59

[2] Cao Bin, Yuan Honglin, Wang Xiaochang, et al. Membrane bioreactor design process parameters [J]. Environmental Engineering, 2004, 22 (5), p.24

[3] Jiang Lan Lan, Zhang Wanli, Hu Bang, et al. Key technology of MBR process membrane system design for urban sewage treatment project [J]. China Water Supply and Drainage, 2011, 27 (20), p.59

[4] Christi Y., Moo-Young M. Improve the performance of airlift reactors. Chemical Engineering 
Process, 1993, 6, p.38

[5] Jiang Lan-lan, Liu Jin and Qian Zhao-yang. Treatment of Rural Domestic Sewage by MBR/Constructed Wetland Process [J]. China Water \& Wastewater. 2010, 26(4), p.127

[6] Chen Yi, Sun Bao-sheng and Zhang Bin. Nitrifying bacteria structure community of different MBR reactor [J]. China Environmental Science. 2010, 30(1), p. 101

[7] Qiu Guang-lei, Song Yong-hui and Zeng Ping. Treatment of Synthetic Bebeerine Wastewater in a Hybrid Upflow Anaerobic Sludge Blanket Reactor (UASB)-Membrane Bioreactor (MBR) System [J]. Research of Environmental Sciences, 2010, 37(7), p. 23

[8] Chen Wei, Wu Di and Zhu Ning-wei. Enhanced Ammonia Removal and Nitrification Rate of A/A/O-MBR Combined Process [J]. Journal of Chongqing Jianzhu University, 2010, 32(4), p. 205

[9] Gao Yong, Hu Rongzheng and Zhu Qing. Experimental Study on Dyeing Wastewater Treatment by Using MBR-Photocatalysis [J]. Technology of Water Treatment, 2010, 36(3), p. 400

[10] Jiang Lan-lan, Hu Bang and Zhang Wan-li. Application of MBR process on wastewater treatment engineering in Taihu Lake Basin [J]. Water \& Wastewater Engineering, 2011, 37(1), p. 305 